\title{
Chapter 14 \\ Surface Nano-structured Coating \\ for Improved Performance of Axial \\ Piston Pumps
}

\section{Antonino Bonanno, Mariarosa Raimondo and Stefano Zapperi}

\begin{abstract}
The work starts from the consideration that most of the power losses in a hydraulic pump is due to frictional losses made by the relative motion between moving parts. This fact is particularly true at low operating velocities, when the hydraulic lift effect must be able to maintain a minimum clearance in meatus to limit the volumetric losses. The potential of structured coatings at nanoscale, with super-hydrophobic and oleophobic characteristics, has never been exploited before in an industrial application. The work studies the potential application of nanocoating on piston slippers surface in a real industrial case. The aim is to develop a new industrial solution to increase the energetic efficiency of hydraulic pump used in earthmoving machines. The proposed solution is investigated using a dedicated test bench, designed to reproduce real working conditions of the pump. The results show a reduction of friction coefficient while changing working pressure and rotation velocity.
\end{abstract}

\subsection{Scientific and Industrial Motivations}

Hydraulic pump efficiency is one of the main concerns in trying to improve the overall efficiency of mobile off-road machines and industrial plants. Axial piston pumps and motors are mainly used in heavy-duty applications; the improvement, albeit marginal, in overall efficiency may significantly impact the global efficiency of the machine. The overall efficiency of energy conversion from fuel to unit of production is as low as $18 \%$ on average, and the largest part of the losses are in the internal combustion engine $(\sim 65 \%)$. The efficiency of axial piston pumps can be

\footnotetext{
A. Bonanno ( $\square)$

CNR-IMAMOTER, Istituto per le Macchine Agricole e Movimento Terra, Ferrara, Italy e-mail: a.bonanno@imamoter.cnr.it

M. Raimondo

CNR-ISTEC, Istituto di Scienza e Tecnologia dei Materiali Ceramici, Faenza, Ravenna, Italy

S. Zapperi

CNR-IENI, Istituto per l'energetica e le Interfasi, Milan, Italy
}

(C) The Author(s) 2019

T. Tolio et al. (eds.), Factories of the Future,

https://doi.org/10.1007/978-3-319-94358-9_14 
improved by means of a surface treatment technology mimicking what in the nature is well known as the Lotus effect. The application of this technology to a real product, with measurable objectives, would represent a result that can be quickly exported to a new generation of industrial products.

In general, structuring of nanoscale surfaces has an impact on their wetting and therefore fluid interaction to obtain high levels of repulsion against water (superhydrophobicity) or oils (oleophobicity) to which they come into contact. Many living organisms in nature have superhydrophobicity or oleophobicity (Lotus leaves, Nephentes plant, etc.), providing a valid chemical-structural model for the production of synthetic surfaces of industrial interest. The literature presents many examples of methodologies, or techniques, for modulating liquid-surface interaction through surface roughness control, which induces the so-called, Wenzel state (partial wettability) to Cassie-Baxter's level that can guarantee high values of contact angle (CA) between liquid and surface and, hence, high repulsion [1]. It is known that a nanometric surface roughness (i.e. nanostructures with appropriate morphology-like pillars, protuberances, etc.) causes that the liquid in contact with the surface is not able to penetrate these discontinuities and is forced to remain on top of them. In this case, the CA between liquid and surface reaches very high values (greater than $150^{\circ}$ ), ensuring very low wettability. CA values also depend on other physical parameters, including surface liquid tension and surface energy of the material, which is in turn related to surface chemistry suitably combined with geometric factors. The technology proposed in $[2,3]$ is able to produce very high CA surfaces (up to $170^{\circ}$ ) and extremely low surface energy $(<1 \mathrm{mN} / \mathrm{m})$ on different types of substrates (ceramics, metals, copper alloys and aluminium).

The aim of this work is to develop a new generation of axial piston pumps by investigating the potential of a novel nanostructured coating, featuring superhydrophobic and oleophobic behaviour that can provide better performance to the interface between lubricated moving parts of the hydraulic pumps and motors. This requires to study at microscopic level the influence of surface roughness on the static or dynamic wetting behaviour of fluids by means of numerical simulations. A key issue is to find the optimal properties in terms of nano-patterning for friction minimization. Therefore it is needed to investigate how and to what extent the superhydrophobicity and oleophobicity of the coatings depends on their specific structure at atomic-scale level. In summary, this work set the following goals:

- Development of a nanostructured coating able to drastically change the fluid surface interaction, specifically reaching a contact angle greater than $150^{\circ}$ for water and $110^{\circ}$ for hydraulic oil, reducing the $\mathrm{CAH}$ (contact angle hysteresis) towards water to values lower than $5^{\circ}$, and reducing the surface energy to values lower than $10 \mathrm{mN} / \mathrm{m}$.

- Reduction of friction coefficient (10/15\% at least) in parts of a hydraulic pump subjected to relative motion.

- Characterization of friction and wear behaviour of the developed coat.

- Development of a computational model of friction and lubrication of confined layer. 
- Development of a hydraulic pump prototype able to work using the nanocoated components.

The chapter is organized as follows. Section 14.2 gives an overview of the state of the art. Section 14.3 presents the steps of the proposed approach to functionalize the surfaces of moving elements. Section 14.4 give the details of the developed methodologies and tools, whereas the experiments and the analysis of the results is addressed in Sect. 14.5. The conclusions are drawn in Sect. 14.6.

\subsection{State of the Art}

The main causes for efficiency reduction in hydraulic components are the lack of adequate drain flow to maintain lubrication between moving parts and the lack of hydrodynamic lift to slippers and delivery port plate [4]. Considering a performance curve of a typical open circuit, variable displacement, axial piston pump, the critical areas can be easily identified (for a given rotational speed) at low delivery pressure. On the other hand, if the rotational speed is changed while keeping fixed the delivery pressure, then it can be demonstrated that low speed conditions are the most critical. Some attempts have been made in the past to overcome these limitations by using ceramic materials [5] or special metallic coatings [6], but state of the art commercial solutions still rely on surface finishing and geometric design. The potential benefits for hydraulic pumps and motors, deriving from the application of the approach proposed in this work, have been studied only recently.

The improvement of the energy performance (friction reduction) of the slipper in axial piston pumps with inclined plate has been addressed by several authors $[4,5,7]$ using experimental measures or through the use of computational fluid dynamics (CFD) codes. Most of these used Reynolds' simplified lubrication or a complete 3D Navier-Stokes approach [8]. All models are based on the existence of an anti-slip bond between fluid and solid. On the contrary, this paper removes such assumptions thanks to the potential of a new surface geometrical configuration that is able to generate a lubrication micro-channel with a slipping length of the same order of magnitude of the geometric interface $[1,4,5]$. The movement of the fluid, after the application of an external force, is influenced by the dynamic behavior of the sliding surfaces. The resistance and lifting forces generated by the fluid are also a function of the dynamic interaction between surface and fluid. There are very few scientific articles presenting and describing these phenomena. Some [5] consider the field of laminar motion, others [7, 8] turbulent conditions. However, among the reported studies, no one applies the concept to real application cases and no existing document links the effect of reduction of friction resistance, by means of coating, to the efficiency of the components.

It is well known from the literature that structural modifications can be induced by controlling the roughness degree of surfaces, so let wetting phenomena moving from an homogeneous state (termed as Wenzel state) to a discontinuous one 
(termed as Cassie-Baxter state), this latter characterizing low wetting conditions [9]. When roughness increases and nanostructures (i.e. pillars, bumps, etc.) develop, fluids droplets falling on a surface are forced to stay on the top of asperities, giving rise to high, stable hydrophobicity which, in some cases where surface chemistry is adequately controlled, can also mean high oleophobicity. The air trapped on the rough surface, in fact, makes the fluid droplet bead up at very high contact angle, which in the case of superhydrophobic surfaces can reach values greater than $150^{\circ}$ or greater than $120^{\circ}$ for oleophobic ones. The modification of chemistry, and hence of surface energy, is also necessary to improve repellence to fluids, especially when the target is the repellence towards oils. Surface energy and surface tension, in fact, are the other two fundamental parameters for phenomena occurring at interfaces of a solid-liquidair system. Due to the quite low values of surface tensions of oils or alkane (much lower for example than water), the design of oleophobic materials requires to lower, as much as possible, the solid surface energy. Oleophobic performance described in the literature $[10,11]$ correspond to surface energy values lower than $10 \mathrm{mN} / \mathrm{m}$. In this context, the preliminary results concerning the technology applied in this work, revealed to be more efficient, producing materials with surface energy lower than 1 $\mathrm{mN} / \mathrm{m}$ (substrates: ceramics, metals or alloys) [12-14].

The new superhydrophobic and oleophobic nano-coated surfaces need to be studied, also, from a computation modelling point of view. The nanoscale properties of boundary lubrication were modelled using large scale molecular dynamics (MD) simulations, in order to obtain a complete multiscale description of the frictional dissipation. MD simulations are really important to estimate the friction and lubrication phenomena, considering the new coated surfaces in a mathematical model of the pump. The numerical boundary lubrication model was obtained performing molecular dynamics (MD) simulations of the frictional properties of lubricant molecules in confined layers with superhydrophobic and oleophobic nanopatterned surfaces. MD simulations follow the dynamics of all atoms in controlled computational experiments where the bulk properties, interface geometries, mechanical loading and interparticle interactions can be varied to explore their effect on friction. MD simulations are a standard methodology, largely exploited in the literature [15-17] to address wetting mechanisms and validate empirical and theoretical models. If the considered system contains enough liquid molecules, MD is indeed in principle capable to provide information about macroscopic parameters such as the density, surface tension, viscosity, flow, static and dynamic contact angle which eventually can be compared with experimental results.

\subsection{Problem Statement and Proposed Approach}

In a common hydraulic piston pump there is a set of parts that are in relative motion (see Fig. 14.1): the pistons move alternately inside the cylinder block, the slippers slide on the swashplate, the cylinder block rotate around his axis and slides on the distribution plate. Herein, the attention is focused on the slippers to reduce the 


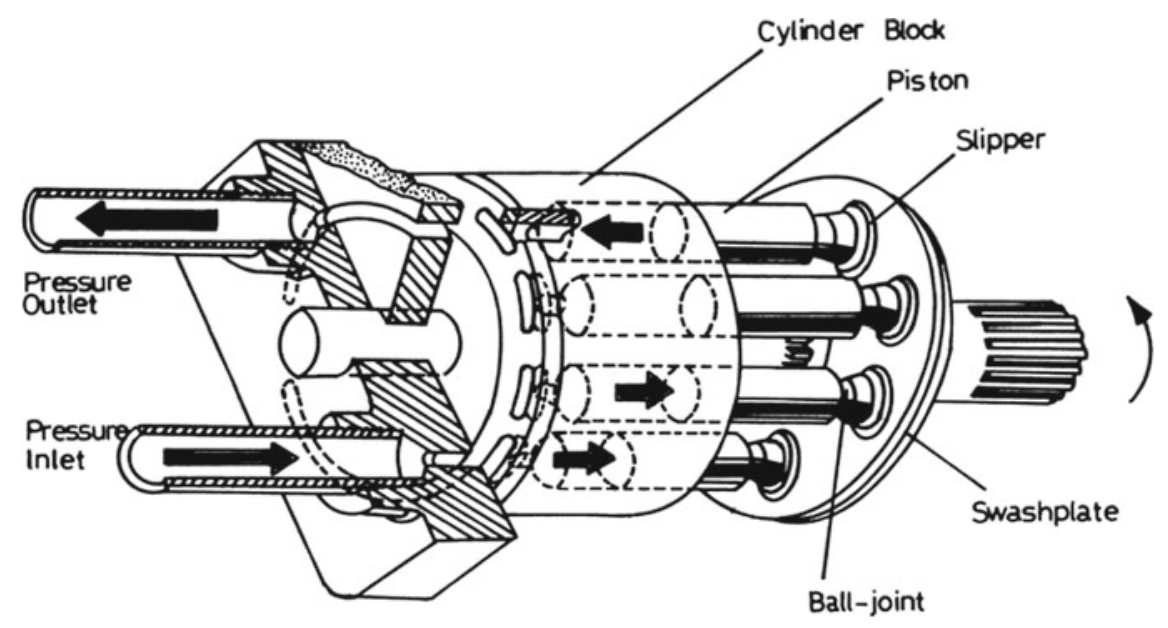

Fig. 14.1 Hydraulic piston pump

possible coating problem (geometry dimension, possible edge phenomena), and also considering the lubrication regime. The lubrication regime that is established between the slipper and the swash plate is hydrostatic, since the high pressure oil, which flows into the oil chamber under the slipper through the shock absorber orifice, generates a hydraulic force able to separate the two elements and allow their movement with low energy expenditure. The design of the slipper is focused on the balance between the hydraulic separation force and the pressure force acting on the slipper. The pressing force acting on the slipper changes continuously during the normal working condition of the pump, thus modifying the equilibrium point between the acting force and the reaction created by the lubricating film. Nevertheless, it is possible to maintain the stability of the whole system thanks to the damping effect created by the orifice. The traditional lubrication theory [18-21] shows that, between the slipper and the swashplate there is a lubricant layer. The velocity profile of this layer shows a velocity zero near the slipper (boundary layer) and a velocity equal to the swash plate linear velocity near it. In this work it was investigated the possibility to reduce (eliminate) the part of the layer where the flow velocity reduces to zero, by coating the slipper with a superhydrophobic/oleophobic layer, which enables the boundary layer to have a velocity different from zero although it moves on a fixed surface.

The problem was faced with a multi-objective approach. The first step (Sect. 14.4.1) was dedicated to the design and synthesis of functional layer, i.e. the design and synthesis of inorganic and hybrid organic-inorganic coatings able to reduce or modulate the wettability of slippers against fluids. In this context, the work was firstly focused on the synthesis of ceramic oxides nanoparticles $\left(\mathrm{Al}_{2} \mathrm{O}_{3}\right.$, $\mathrm{ZrO}_{2}$, etc.) in the form of stable colloids or nanosuspensions in different media (i.e. alcohols or water), getting a high control degree on phases, particle size and composition. Also specific synthetic approaches were developed (i.e. sol-gel), involving 
particle nucleation directly from precursors present into the fluid, in the meantime avoiding particle precipitation and ensuring a better control over physical variables of suspensions.

The possibility to apply the developed oxide on the slipper was addressed in the second step of the approach, by assessing the adaptation of deposition techniques to the to specific piece design (Sects. 14.4.2, 14.5.1). In this context, coating deposition by simple immersion techniques, such as dip-coating or spin-coating in controlled conditions, was investigated to produce homogeneous functional surfaces, to easily control the layer thickness in the order of nanometers, and to promote the formation of micro- and nanostructures.

The assessment of tribological performance of the obtained samples was the third step of the approach (Sects. 14.4.3, 14.5.2). The new surface treatment technology should assure the reduction of friction but, at the same time, the pump ability to work in the expected operative conditions, so it is necessary to have a complete tribological and mechanical characterization of functional layers. A non-contact profilometry, a detector of acoustic emission and a nanoindentator were used for this activity. The first instrument was used to obtain the topography of the metals and alloys coated. The acoustic detector gave the possibility to know the strength between the substrate and nanostructured coating. The second instrument allowed to evaluate the coating hardness of the surface layers.

The hydrostatic lubrication uses the fluid pressure to separate two surfaces that have a sliding motion. If the lubricating film thickness does not suffice to separate the sliding surfaces completely then wear takes place. In order to know how, the new superhydrophobic and oleophobic surface modified the hydrodynamic lubrication in comparison to the current slipper, a torque meter was used to evaluate the absorbed torque considering the different lubricating condition (low and high pressure, low and high velocity). Pressure sensor and flow meter were used to know how much flow is necessary to ensure a good lubrication conditions at different pressure. These pieces of information are relevant from a fluid power point of view, due to the possibility to evaluate the oil flow used to maintain the hydrostatic lubrication. At the same time the oil pressure and the flow necessary to guarantee the meatus using the nanocoated surface were measured and compared to that measured using the traditional surface. In addition, the absorbed torque was acquired to have information about the mechanical efficiency.

The final part of the work was dedicated to the MD simulations (Sects. 14.4.4, 14.5.3) to study, also from the computational point of view, the lubrication conditions obtained using the nanosctructured coating. The problem was faced by employing different available simulation packages and tools to build a powerful and efficient computational framework specifically aimed at performing extended MD simulations and hybrid multiscale modelling. 


\subsection{Developed Technologies, Methodologies and Tools}

\subsubsection{Design and Synthesis of Functional Layer}

The first part of the work was focused on the sol-gel synthesis of ceramic oxide nanoparticles suspended in different solvents (water and alcohols) which can be used to create coatings able to modify the wettability of materials. Superhydrophobic (water repellent) and superoleophobic (repellent towards fluids with a lower surface tension than water) surfaces on different materials, particularly on ceramics, metals and alloys, were developed starting from two patents [2, 3]. Moving from the evergrowing literature [15] the activity focused on the ability to produce precursors of functional coatings through water-phase synthesis (instead of an alcohol-phase synthesis) in order to reduce environmental impact and move towards a more favourable transfer to the industrial scale in terms of costs and safety.

The precursor of the ceramic oxide is aluminium tri-sec-butoxide; in water solvent, first it undergoes hydrolysis, then it forms Al-O-Al bonds during a peptization process. To avoid a too fast hydrolysis of the butoxide with $\mathrm{Al}(\mathrm{OH})_{3}$ precipitation, a chelating agent was dissolved in water (ethyl acetoacetate) before adding the butoxide; the chelating agent substitutes the butoxide, slowing down the hydrolysis. In order to obtain a good granulometric distribution of the suspension, which has to stay below $100 \mathrm{~nm}$ to lead to the formation of nanoparticles and avoid aggregates, a $\mathrm{HNO}_{3}$ solution is gradually added to the mixture; protons bond to and charge the surface of forming particles, stabilizing them and avoiding aggregation. After $24 \mathrm{~h}$ at $70{ }^{\circ} \mathrm{C}$, a transparent sol is obtained $(\mathrm{pH}=3.64)$. A scheme of the reaction is reported in Fig. 14.2a.

\subsubsection{Slippers Coating}

After the sol preparation, the activities were focused on the slippers coating. Before the functionalization, the samples were sandblasted to create a micro-scale roughness which promotes adhesion by the coating and provides better mechanical resistance [16]. Usually, sandblasted samples show roughness of about 4-5 $\mu$. Figure 14.2b shows the dip coated used to coat samples with different morphology and size. The methodology is easily transferrable to industrial scale. The slipper samples were dipped in the sol in two different configurations: with horizontal axis and with vertical axis.

The dipping conditions of the skid in the sol were the following:

- Dipping speed: $2 \mathrm{~mm} / \mathrm{s}$.

- Time standing still in the sol: $5 \mathrm{~s}$.

- Withdrawing speed: $2 \mathrm{~mm} / \mathrm{s}$.

After emersion of the samples, the coatings required the following treatments: 

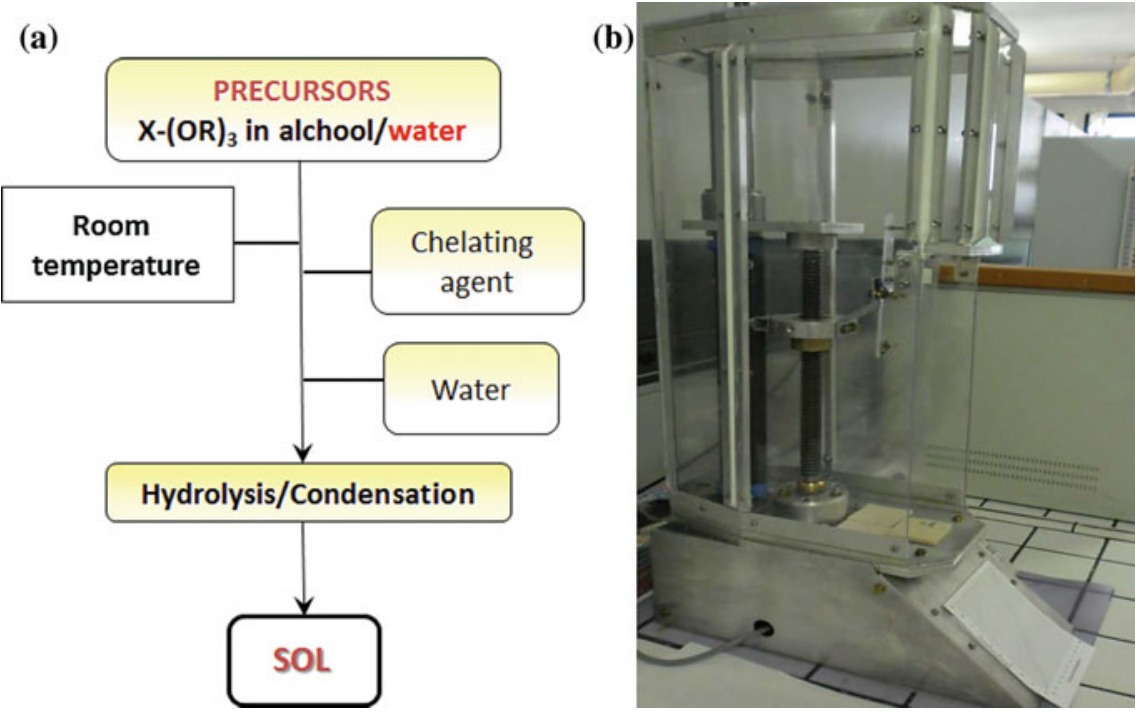

Fig. 14.2 a Sol-gel synthesis methodology; b dip coater

- First thermal treatment: $400{ }^{\circ} \mathrm{C}$ for $60 \mathrm{~min}$ in muffle furnace.

- Boiling in water for $30 \mathrm{~min}$ to create the so-called flower-like boehmite $\mathrm{AlO}(\mathrm{OH})$ nanostructure.

- Second thermal treatment: $400{ }^{\circ} \mathrm{C}$ for $10 \mathrm{~min}$ in muffle furnace to reinforce boehmite film.

- Dipping in a fluoroalkylsilane solution (FAS-Dynasilan SIVO Clear EC di Evonik) (withdrawing speed $2 \mathrm{~mm} / \mathrm{s}$, time standing $2 \mathrm{~min}$ ) to lower surface energy.

- Final thermal treatment at $150^{\circ} \mathrm{C}$ for $30 \mathrm{~min}$ to allow crosslinking by the fluorinated layer and reinforce the film.

All experimental settings were optimized to obtain the best wettability performance for the coatings. Furthermore, it was verified that the samples were dimensionally stable at the temperatures used during thermal treatments. Functionalized samples were then characterized in terms of wettability with water and lubricant Arnica 46, representative of the oils used in axial pumps.

\subsubsection{Slipper Test Bench}

A slipper test bench was designed and realized to test the coated slippers without needing to use a pump. Figure 14.3 shows the test bench designed to measure the friction reduction obtained using the superhydrophobic/oleophobic layer. It uses an inverse working principle compared to the real hydraulic piston pump. In fact, in the pump, the piston block rotates, moved by the transmission axle, dragging the piston, 


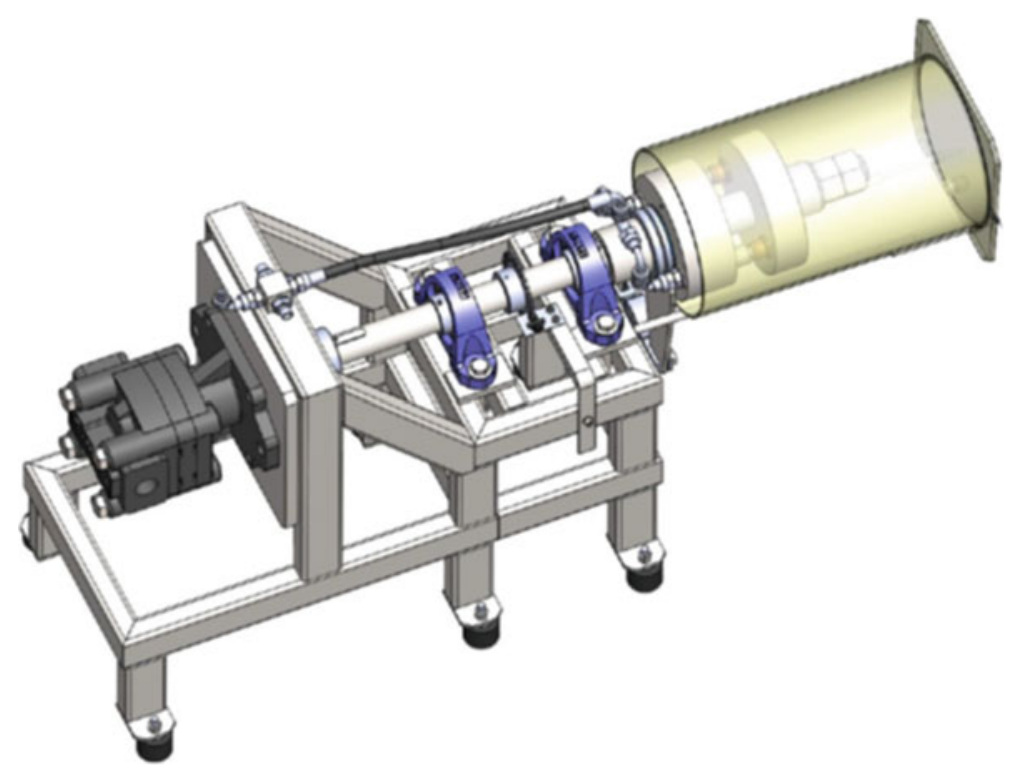

Fig. 14.3 Slipper test bench

and consequently the slippers, in his rotation. The slippers slip on the plate, which is fixed. In the test bench, due to realization problems, the principle was inverted and the plate rotates while the slippers have only the possibility to move axially on the plate. From a kinematic point of view, the movement is the same: a relative sliding between the slippers and the plate.

The frame is made by tubular profile structural steel (FE 360B). The elastic coupling, constituted by a double wheel hub and a star in polymeric material, allows the transfer of mechanical power to load. The dimensions and materials have been chosen according to the geometric dimensions of the shaft and taking in consideration that the kinematic system is subjected to a mechanical locking. The transmission shaft was made of $39 \mathrm{NiCrMo} 3$ tempered steel, thus guaranteeing good tenacity (typical in the tuning phase and in the case of accidental failure). The ball bearings mounted on rigid supports have been chosen in order to guarantee a maximum rotating speed of $3000 \mathrm{rpm}$. The advantages of this configuration are many: the possibility to see the slipper behaviour, the possibility to measure the friction torque and then the friction coefficient, the possibility to easily change the tested prototype without disassemble a complete pump. The results of the tests performed using the designed test bench are showed in the next section. 


\subsubsection{Simulation}

Another important task is the study, at a microscopic level, of the influence of surface roughness on the static/dynamic wetting behaviour of fluids by means of numerical simulations. Our aim was to understand how and to what extent the superhydrophobicity/oleophobicity of the coatings depends on their specific structure at atomic-scale level. The MD (molecular dynamic) study was approached within the framework of a basic standard solid-fluid model, which allows us to outline and understand the main features of the fluid interaction with a nano-patterned surface in a simple and effective way. We modelled solid-solid and solid-fluid interaction with the Lennard-Jones pair potential reported in (14.1) which is suitable for neutral atoms or molecules and is composed of a steep short-range repulsive term and a smoother long-range attractive one (van der Waals type).

$$
E_{L J}=\left\{\begin{array}{lr}
4 \varepsilon\left[\left(\frac{\sigma}{r}\right)^{12}-\left(\frac{\sigma}{r}\right)^{6}\right] & r<r_{c} \\
0 & r>r_{c}
\end{array}\right.
$$

The relevant quantities are the depth of the potential well $(\varepsilon)$, the finite distance at which the interparticle potential is zero $(\sigma)$, the distance between the particles $(\mathrm{r})$, and the cut-off distance at which the potential vanishes $\left(\mathrm{r}_{\mathrm{c}}\right)$. We have two sets of parameters, one for the solid-fluid interactions and one for the fluid-fluid interactions, but in the following pictures we used dimensionless units and we just had to deal with two parameters $\varepsilon_{*}=\varepsilon_{\mathrm{FS} /} \varepsilon_{\mathrm{FF}}$ and $\varepsilon \sigma_{*}=\sigma_{\mathrm{FS}} / \sigma_{\mathrm{FF}}$. In this model the $\varepsilon_{*}$ parameter represents an effective interaction and accounts for all the aspects of the solid-fluid interactions due to material properties and chemical treatments. This is the parameter to be varied to outline in general the role of surface chemical condition in frictional dissipation. The role of nanopatterning from a geometrical point of view is instead studied by varying the roughness of the solid surfaces, i.e. their geometrical texture in terms of vertical deviations from the flat condition (Fig. 14.4).

We implemented also MD simulations of a fluid confined between walls with rough surfaces in order to study how roughness affects the friction at the boundaries. A shear (Couette) flow in the channel was induced by moving the upper wall with velocity $\mathrm{v}_{0}$ and the lower wall with velocity $-\mathrm{v}_{0}$. We vary the $\mathrm{L}_{\mathrm{J}}$ parameters in a range such that the velocity profile is always $\approx$ linear. In the presence of nanostructured coatings, the interaction between fluid and wall could be described as sliding, i.e. a situation in which the value of the tangential component of the speed seems to be different from that of the solid surface. This behaviour is described, in the simplest way, assuming that the tangential force per unit area exerted on the solid surface is proportional to the sliding speed, i.e. $\sigma_{x z}=k v_{\text {slip }}$ where $x$ is the direction of the flow and $z$ the orthogonal one. Combining this with the constitutive equation for the bulk Newtonian fluid $\sigma_{x z}=\eta \vartheta_{z} v_{x}$ one gets the so-called (scalar) Navier boundary condition $v_{\text {slip }}=\eta / k \vartheta_{z} v_{x} \equiv \delta \eta \vartheta_{z} v_{x}$. The last equality defines the slip length $\delta=\eta / k$ which represents the distance inside the solid to which the velocity 

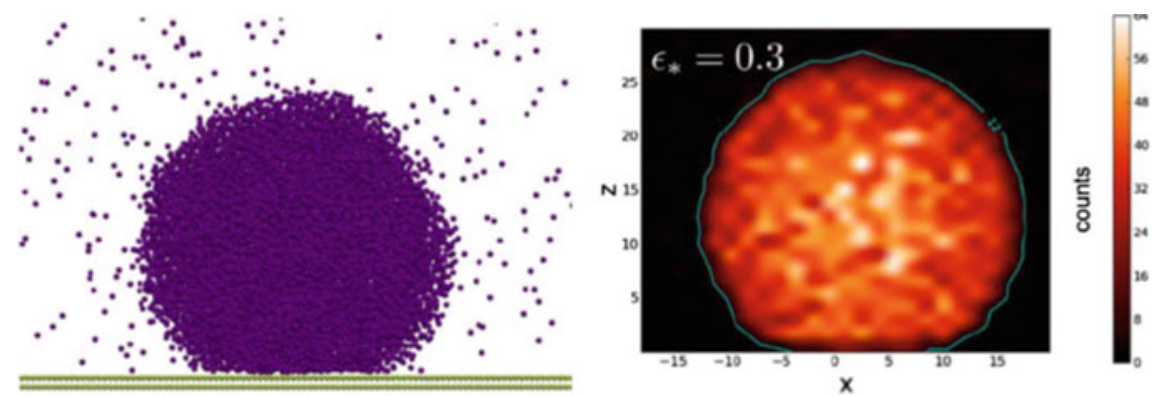

(1) $2 d$ density plot of the drop atoms (e.g. $x-z$ and $y-z$ planes) and contour plot of isodensity lines
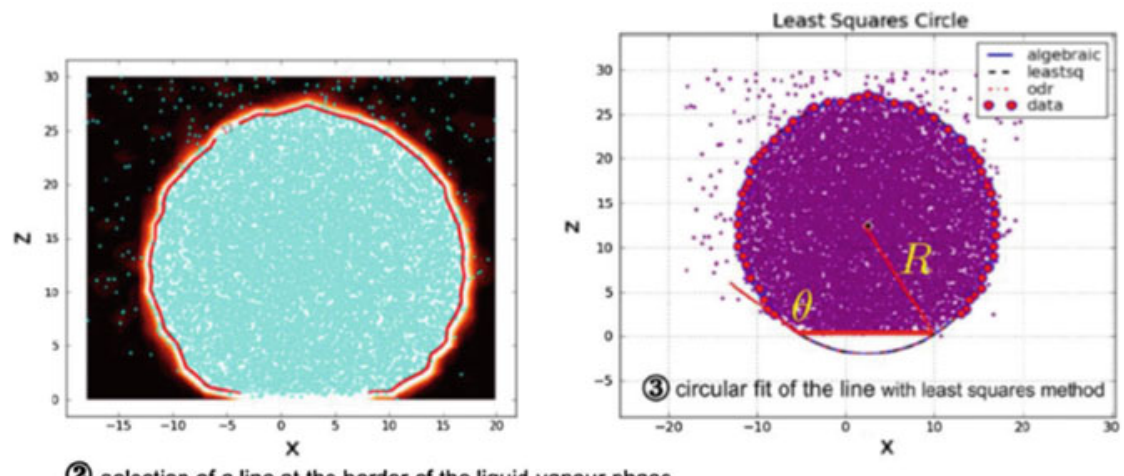

selection of a line at the border of the liquid-vapour phase

Fig. 14.4 Summary of the procedure adopted to extract the contact angle from MD results

has to be extrapolated to reach zero. This slip length represents a measure of the friction of the fluid at the solid walls and is thus influenced by the surface roughness, which is expected to reduce friction in the same fashion as it reduces the contact angle. These results basically confirm what we already found in the case of the static contact angle and conform to the existing literature: the rougher the surface (at fixed interaction potential) the better the slippage, i.e. less friction.

\subsection{Experiments}

Several specimens were developed during the work. The state of the art shows the possibility to coat laboratory samples having flat (or approximately flat) surface, without edges, undercut, or abrupt change of section. However, in this work the attention was focused on a real industrial component, i.e. the slipper of a hydraulic pump. Therefore, the greater part of specimens are functionalized (coated) slippers which were developed to test and investigate the superhydrophobic/oleophobic layer performance, the different coating (with and without ceramic substrate), and the 
1
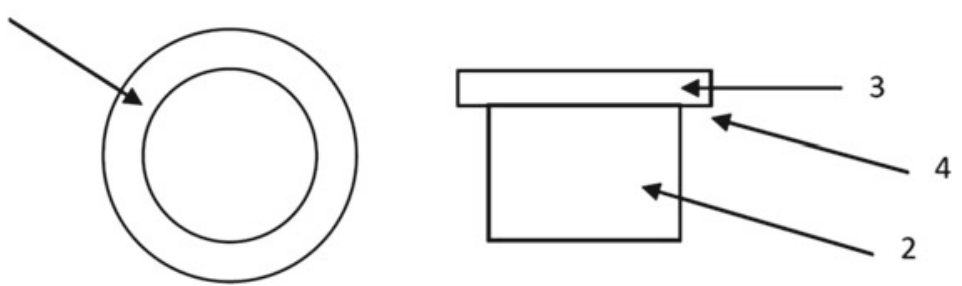

Fig. 14.5 Mapping of different zones were the CA characterization were performed

different slippers geometries. Furthermore, a complete pump was realized and tested in a dedicated test bench.

The slipper shows a complex surface from the coating perspective (see Fig. 14.5).

\subsubsection{Fluid Surface Interaction}

The first prototypes were developed starting from the slipper geometry actually used in the industrial hydraulic piston pump. No modification was introduced except the application of nanostructured coating on the sliding slipper surface. Eight couples of functionalized slippers were made by coating the sliding surface.

After the functionalization of the surfaces, the performance was characterized by measuring the WCA (water contact angle), the CAH (contact angle hysteresis), the SE (surface energy), and the OCA (oil contact angle). The results obtained can be summarized as follow:

- The mean WCA of standard sample, measured in zone 1 and 2 were respectively $74.8^{\circ} \pm 1.0^{\circ}$ and $88.9^{\circ} \pm 1.1^{\circ}$.

- The mean WCA of functionalized samples, coated in horizontal position, was $142.6^{\circ} \pm 2.6^{\circ}$ degree in zone $1,122.0^{\circ} \pm 5.1^{\circ}$ in zone $2,120.8^{\circ} \pm 2.7^{\circ}$ in zone 3 and $124.4^{\circ} \pm 3.0^{\circ}$ in zone 4 .

- The mean WCA of functionalized samples, coated in vertical position, was $158.8^{\circ} \pm 2.8^{\circ}$ degree in zone $1,123.5^{\circ} \pm 3.0^{\circ}$ in zone $2,134.7^{\circ} \pm 1.6^{\circ}$ in zone 3 and $112.2^{\circ} \pm 1.0^{\circ}$ in zone 4 .

The results demonstrate that the nanostructured coating, developed as explained in the previous section, is able to change the surface wettability reaching contact angle typical of superhydrophobic layer $\left(\mathrm{CA}>150^{\circ}\right)$. The vertical position is more efficient in term of coating performances. The developed methodology is able to drastically modify the slipper working surface (zone 1 in Fig. 14.5) contact angle from $74.8^{\circ}$ to $158.8^{\circ}$. The contact angle hysteresis $(\mathrm{CAH})$, using water as the measurement fluid, was measured by needle technique (droplet volume $2 \mu \mathrm{L}$ ). The smaller the hysteresis value, the better the ability of a surface to make a drop slip (better dewetting properties [1]). CAH values measured on treated samples were: 
- $4.54^{\circ} \pm 2.04^{\circ}$ for horizontal coating configuration

- $1.90^{\circ} \pm 1.50^{\circ}$ for vertical coating configuration.

The sample dipped with vertical axis proved to be more performing also in terms of hysteresis. The static contact angle was measured by diiodiomethane. Evaluations were carried out on zones 1 and 2 both on the treated samples and on the sanded sample. Using the Owens-Wendt-Rabel-Kaelble algorithm it is possible to calculate the surface energy in the different points of the analysed samples. The measurements are made by comparing the results obtained with diiodiomethane (surface tension 50.8 $\mathrm{mN} / \mathrm{m}$ ) with those obtained with water. The results obtained showed the efficiency of nanostructured coating: the SE of coated sample (vertical coating configuration) results $0.47 \pm 0.05(\mathrm{mN} / \mathrm{m})$ against a surface energy of $38.87 \pm 1.00(\mathrm{mN} / \mathrm{m})$ measured for the standard sample. The comparison of the results obtained on the coated slipper with those obtained from the standard sample shows that the surface energy is reduced to values close to zero. Of particular relevance is the fact that the lowest SE value $(0.47 \mathrm{mN} / \mathrm{m})$ was measured on zone 1 of the sample, the working face normally used by the slipper. Low surface energy is a fundamental parameter, together with surface nanostructure, to obtain oleophobicity $[9,10,22]$, which is the repellence towards low surface tension liquids (at least lower than water). The CA measurement performed considering ARNICA 46 hydraulic oil as fluid showed again the high repellence obtained thanks to the nanostructured coating:

- CA with ARNICA 46, standard sample: $16.7^{\circ} \pm 2.3^{\circ}$

- CA with ARNICA 46, functionalized sample: $123.8^{\circ} \pm 11.8^{\circ}$.

\subsubsection{Tribological Performance}

The following tests were performed to assess the tribological performance:

- The scratch test in order to determine the mechanical strength adhesion, intrinsic cohesion of coating. The tests were performed with a CSM Revtest machine, applying a progressive normal load from 1 to $20 \mathrm{~N}$. The total length of scratch was $5 \mathrm{~mm}$, the indenter speed $10 \mathrm{~mm} / \mathrm{min}$.

- The pin on disc test in order to evaluate the wear and friction characteristics of slippers both in dry and lubricated environments. This test was performed with a CSM tribometer. The test conditions were: load $10 \mathrm{~N}$, speed $20 \mathrm{~cm} / \mathrm{s}$, room temperature, pin $18 \mathrm{MnCr} 5$, pin diameter $2.4 \mathrm{~mm}$, laps 5000 .

- The surface analysis by means of a non-contact profilometer Taylor Hobson Talysurf CCI $3000 \AA$.

A total of eight pairs of samples were tested: 2 standard (sand blasted and not sand blasted) and 6 coated samples. The coated slipper shows quite the same average roughness $(1.5-2.5 \mu \mathrm{m})$ with only one that shows a lowest surface roughness $(\approx 0.8 \mu \mathrm{m})$. 


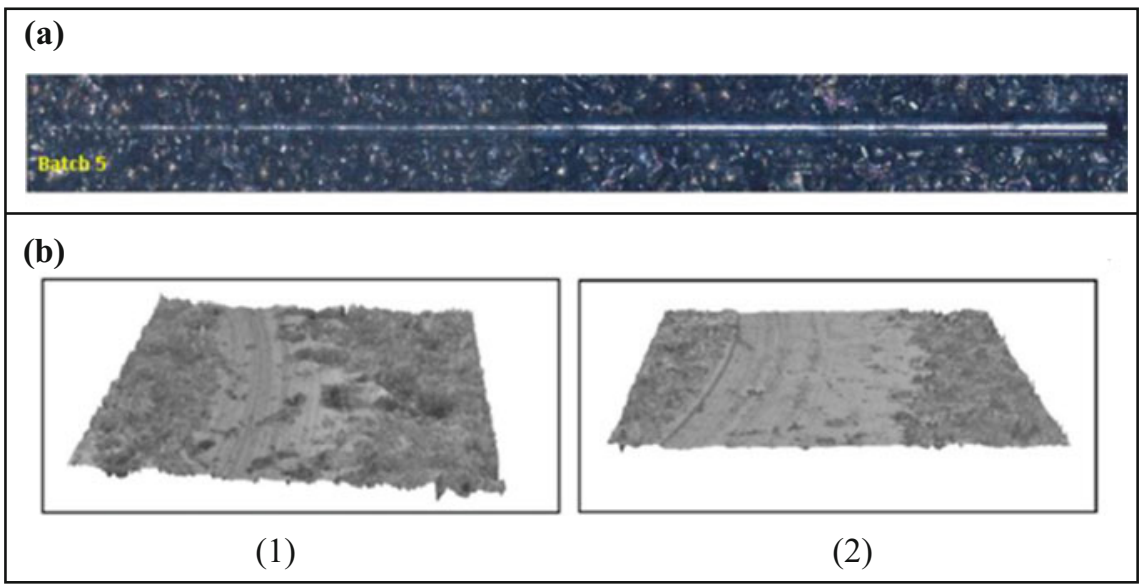

Fig. 14.6 a Scratch track of slipper coated with batch 5; b Wear test tridimensional profiles (1) batch 4 ; (2) batch 5

As for the adhesion results, no substantial differences, in terms of cracks typologies were found between the functionalized slippers. Nevertheless, comparing the acoustic emission, a lower value was found for the batch 1 and 6 . This phenomenon suggests that lower microcraks appeared along the scratch channel.

The lubricant reduces the friction coefficient in all cases:

- WET TEST: average friction coefficient $0.145 \pm 0.035$

- DRY TEST: average friction coefficient $0.233 \pm 0.072$.

As for the surface functionalization, it does not reduce the friction coefficient. However, it must be stressed that the test cannot simulate a hydrostatic lubrication condition, as in hydraulic piston pump. An increment of the wear resistance is observed for the slippers coated with batch 2, 4 and 5. In fact, at the end of the tribological test, it was difficult to measure the track depth. SEM-EDS analysis supports tribological results. In Fig. 14.6 a summary of results is showed.

Different pairs of standard and functionalized samples were tested in a dedicated test bench, considering a constant rotation velocity $(700 \mathrm{rpm})$ and different pressures from 10 to 50 bar. Figure 14.7 shows the mean friction coefficient both for standard and functionalized samples. The friction coefficient reduction is between the $40 \%$ (at lower pressure) and 25\% (at higher pressure),

Other six pair prototypes were made to test the ceramic substrate influence on the slipper behaviour. In fact, it is possible to coat the slipper with fluoroalkylsilane solution (FAS-Dynasilan SIVO Clear EC di Evonik), to reduce the surface energy, without performing a complete surface functionalization (ceramic substrate +FAS coating). The coating with only FAS without the ceramic substrate, although giving comparable results in term of friction reduction in a normal (not endurance) test, do not give as good performance in term of duration, being abraded after $20 \mathrm{~h}$. 


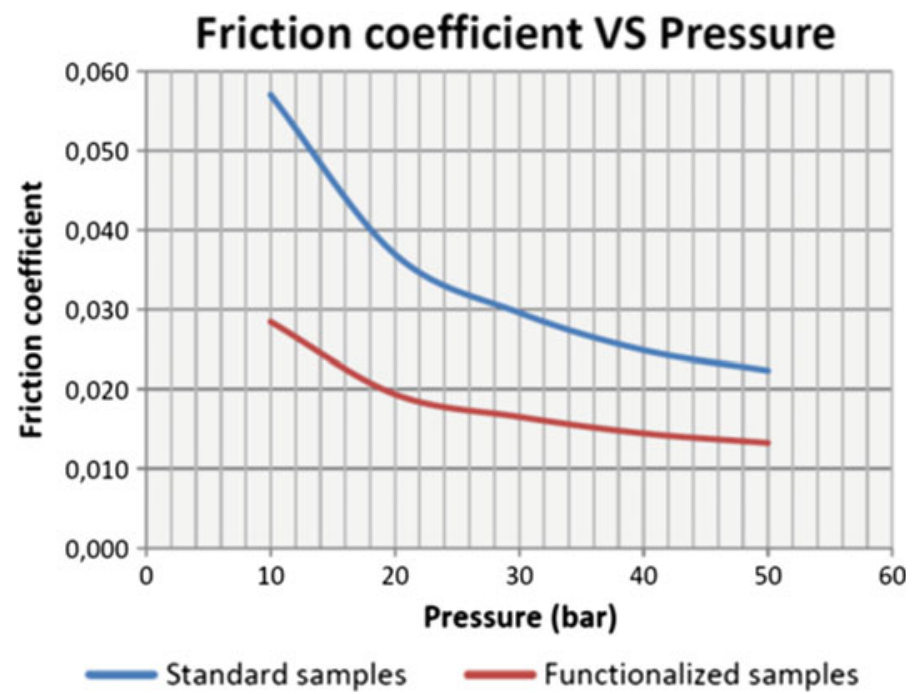

Fig. 14.7 Mean friction coefficient for standard (blue curve) and functionalized (red curve) samples

\subsubsection{Testing the Computational Model}

We started by analysing the static wetting mechanism and simulating liquid drops at equilibrium on different surfaces. To extract the contact angle $\theta$ from the MD result we adopted a simple fitting procedure of the density profile of the drop at equilibrium (Fig. 14.4). We then considered also a dynamical framework, with simulation of a liquid between moving flat/rough walls. We considered the influence on the contact angle due to geometrical nano-scale effects and implemented MD simulation with rough surfaces. We generated randomly rough surfaces with controlled root-meansquare roughness and correlation length and look at the variation of the contact angle as a function of roughness at fixed interaction strength $\epsilon$. Figure 14.8 shows snapshots of drops at equilibrium for different values of the surface roughness compared to the flat case: the key feature is that the contact angle increases for increasing roughness. This corresponds to the well-known fact that surface roughness benefits the fluidrepellent properties when they are present [16, 17, 23].

Figure 14.9 shows the curves representing the contact angle for different surface roughness. It can be noticed that the contact angle increases for increasing roughness.

Regarding the MD simulations of a fluid confined between walls with rough surfaces, we obtained some velocity profiles (not showed) resulting from our simulation for varying roughness. These results confirm that the rougher the surface (at fixed interaction potential), the better is the slippage, i.e. less friction. To study the role of surface conditions in frictional dissipation from a more chemical point of view, we implemented simulations of the static contact angle on a flat surface and we looked its variation as a function of the strength of the solid-fluid interaction. The 


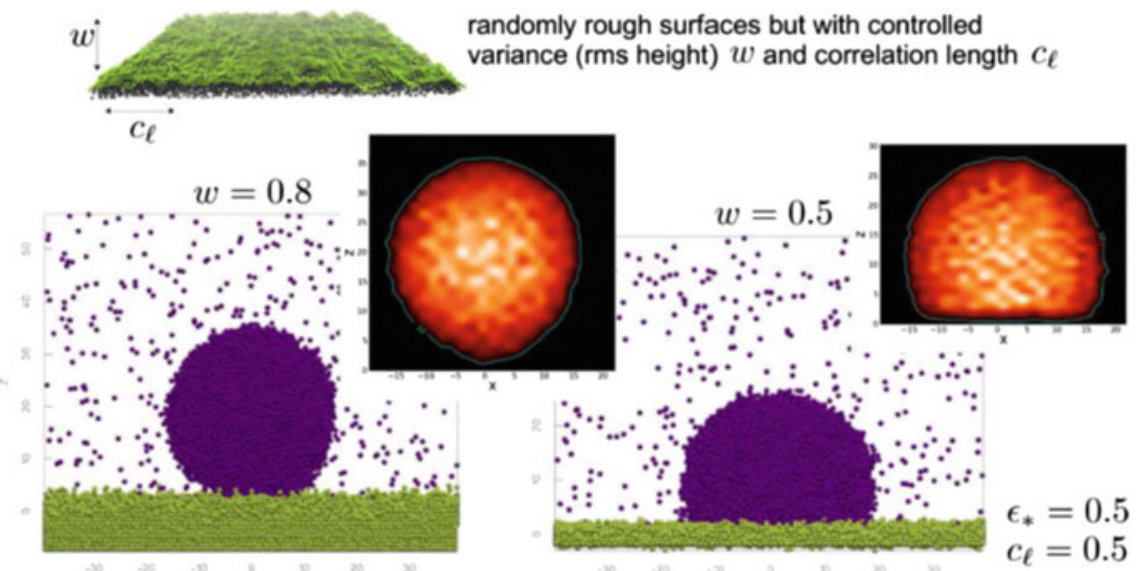

Fig. 14.8 Variation of the contact angle in the presence of a rough surface. A randomly rough surface is generated with controlled root-mean-square roughness $w$ and correlation length $c \ell$ (top). For fixed interaction strength $\epsilon$, we then look at the difference in the contact angle for different values of $\mathrm{w}$, in this example $\mathrm{w}=0.8$ (left) and $\mathrm{w}=0.5$ (right)

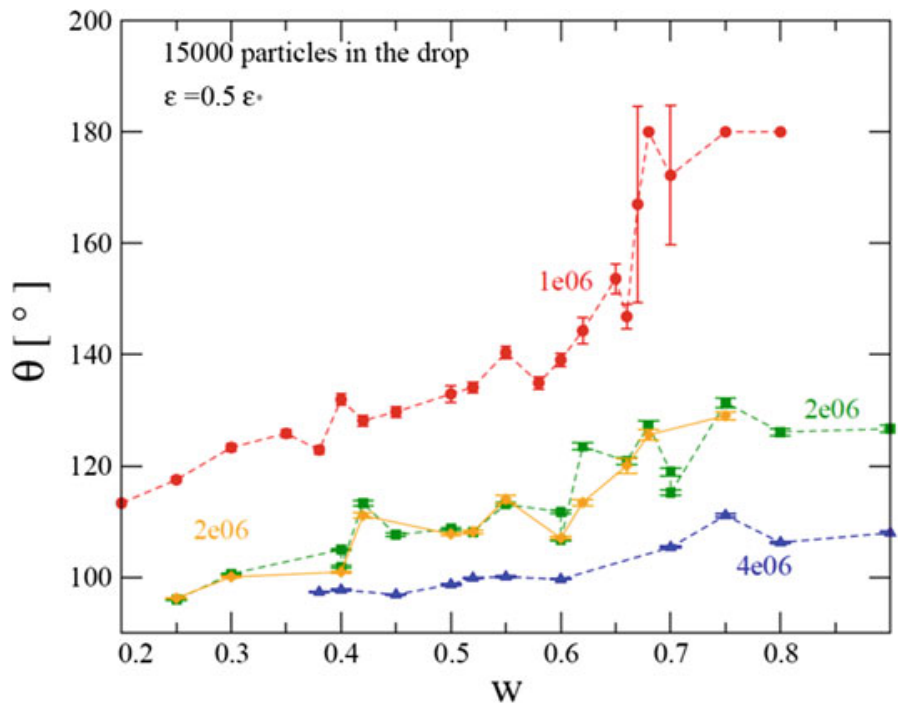

Fig. 14.9 Contact angle on a rough surface as a function of the roughness $w$ for $\epsilon^{*}=0.5$. Different curves correspond to different equilibration times

results obtained show that contact angle is inversely proportional to the strength of the potential. Superhydrophobic coatings would thus be efficiently simulated using the proposed methodology. 


\subsubsection{Testing the Hydraulic Pump}

Finally, a prototype of hydraulic piston pump was realized, starting from a commercial pump (HP P8 made by Hp-Hydraulic, Bondioli \& Pavesi Group) installing functionalized slippers instead of normal one. The pump was tested in a dedicated test bench working in closed circuit. Contrary to the expectations, the results are in contrast to that obtained in the slipper dedicated test bench because the standard slippers work better than functionalized ones. This is probably due to the fact that the slippers, mounted on the pump, are subjected to working conditions that we were not able to reproduce during the test performed in the dedicated test bench. In order to investigate this last phenomenon, other slipper prototypes were made considering geometrical modification.

The results obtained have opened a broad series of questions in which the pump manufacturer has been involved.

\subsection{Conclusions and Future Research}

This work investigated the potential of surface nano-coating of the piston slippers surface, with the aim of achieving better energy efficiency in a real industrial component by means of a drastic reduction of the friction coefficient. We have designed and synthesized a functional layer, obtaining a good granulometric distribution of the suspension [24-28]. We have demonstrated the feasibility of the coating processes and the adaptability of deposition techniques to the hydraulic piston pump slippers (Sect. 14.5.1). Thanks to the nanostructured coating it was possible to obtain, with water, a contact angle of $158.8 \pm 2.8^{\circ}$ (superhydrophobic behaviour), whereas with oil the contact angle is $123.8 \pm 11.8^{\circ}$ (oleophobic behaviour), where the standard slipper show a contact angle of only $16.7 \pm 2.3^{\circ}$ (oleophilic behaviour). The samples where characterized from a tribological point of view (Sect. 14.5.2). Furthermore, wear analysis of the coatings, with particular focus on the relationship between coatings features and wear mechanisms (via SEM-EDS), have been done. Considering the adhesion results, no substantial differences, in terms of cracks typologies were found between the functionalized slippers. The lubricant reduces the friction coefficient in all cases. As for the surface functionalization, it does not reduce the friction coefficient. However, it has to be stressed that the pin-on-disc test cannot simulate a hydrostatic lubrication condition, as we have in the real pump.

The influence of the new superhydrophobic and oleophobic surface on the hydrostatic lubrication was investigated thanks to a dedicated test rig. The results [29-31] showed that, using the functionalized oleophobic coating, is possible to have a friction coefficient reduction from 20 to $30 \%$, in function of the different working condition.

The last part of the work was dedicated to the influence on the contact angle due to geometrical nano-scale effects implementing a MD simulation with rough surfaces (Sect. 14.5.3). The key results obtained were: 
- the contact angle increases for increasing roughness;

- the rougher the surface (at fixed interaction potential) the better the slippage, i.e. less friction;

- stronger fluid-solid interaction (at fixed surface roughness) means less slippage, i.e. more friction.

All these results confirm the literature, demonstrating the performed analysis validity. Future developments will be focused on the investigation of the possibility to apply the same coating on other pump component and to overcome the problems faced during this project, especially those related to the coating wearing.

Finally, the exploitation of the scientific results led to the submission and approval of a research project titled "ERCOLE on APP_Energy Recovery Coatings Originating Lubrication Effects on Axial Piston Pumps" funded under the call of Regione Emilia Romagna dedicated to the factories involved in the seismic events of 2012 (art. 12 DL 74/2012). The project was focused on antifriction solutions (not only oleophobic coating) to improve the efficiency of hydraulic piston pump.

Acknowledgements This work has been funded by the Italian Ministry of Education, Universities and Research (MIUR) under the Flagship Project "Factories of the Future - Italy" (Progetto Bandiera "La Fabbrica del Futuro") [32], Sottoprogetto 1, research projects "Surface Nano-structured Coating for Improved Performance of Axial Piston Pumps" (SNAPP) and "Axial Piston Pump Prototype Assembled with Oleophobic Surfaces Components" (APPOS).

The authors express their gratitude to the following researcher, for the precious and effective collaboration, without which all this work would not be possible: Eng. Giuseppe Rizzo, Eng. Giorgio Paolo Massarotti, Eng. Luca Pastorello, Dr. Maria Giulia Faga, Dr. Giovanna Gautier, Eng Massimo Martelli, Eng. Pietro Marani, Dr. Federico Veronesi, Dr. Magda Blosi, Dr Aurora Caldarelli, Dr. Giulio Boveri, Mrs. Guia Guarini, Dr. Carlotta Negri.

The authors would like to thank HP Hydraulic S.p.A. for its indispensable technical and methodological contribution, gaving the necessary support to perform all the tests and to develop the different prototypes.

This work would not have been possible without the foresight of Prof. Roberto Paoluzzi, in memory of whom this paper was written.

\section{References}

1. Rothstein J (2010) Slip on superhydrophobic surfaces. Annu Rev Fluid Mech (42):89-109. https://doi.org/10.1146/annurev-fluid-121108-145558

2. Rimondo M, Blosi M, Bezzi F, Mingazzini C (2011) Metodo per il Trattamento di Superfici Ceramiche per Conferire alle Stesse una Elevata Idrofobicità e Oleofobicità. Patent RM2011A000104

3. Rimondo M, Blosi M, Bezzi F, Mingazzini C (2012) Metodo di Trattamento di Superfici Metalliche per Conferire alle Stesse una Elevata Idrofobicità ed Oleofobicità. Patent RM2012A000291

4. Manringh N, Wray C, Dong Z (2004) Experimental studies on the performance of slipper bearings within axial-piston pumps. J Tribol 126(3):511-518

5. Kumar S, Bergada JM, Watton J (2009) Axial piston pump grooved slipper analysis by CFD simulation of three-dimensional NVS equation in cylindrical coordinates. Comput Fluids 38(3):648-663. https://doi.org/10.1016/j.compfluid.2008.06.007 
6. Tominaga T, Yamamoto K, Matsumoto O, Hashiba M (1998) Swash plate type axial piston pump. US Patent US005809863A

7. Iwatsu R, Hyun JM, Kuwahara K (1993) Numerical simulation of three dimensional flow in a cubic cavity with an oscillating lid. ASME J Fluid Eng 115:680-686

8. Yao H, Cooper RK, Raghunathan S (2004) Numerical simulation of incompressible laminar flow over three-dimensional rectangular cavities. J Fluid Eng 126(6):919-927

9. Liu K, Yao X, Jiang L (2010) Recent developments in bio-inspired special wettability. Chem Soc Rev 39:3240-3255. https://doi.org/10.1039/b917112f

10. Tuteja A, Choi W, Ma M, Mabry J, Mazzella S, Rutledge G, McKinley G, Cohen R (2007) Designing superoleophobic surfaces. Science 318:1618-1622. https://doi.org/10.1126/science. 1148326

11. Bhushan B (2011) Biomimetics inspired surfaces for drag reduction and oleophobicity/philicity. J Nanotech 2:66-68. https://doi.org/10.3762/bjnano.2.9

12. Raimondo M, (2012) Making super-hydrophobic building materials: static and dynamic behavior of nanostructured surface. In: IV ICC4-International Ceramic Conference, Chicago (USA), July $14-19$

13. Savoy E, Escobedo F (2012) Molecular simulations of wetting of a rough surface by an oily fluid: effect of topology, chemistry, and droplet size on wetting transition rates. Langmuir 28(7):3412-3419. https://doi.org/10.1021/la203921h

14. Niavarani A, Priezjev N (2010) Modeling the combined effect of surface roughness and shear rate on slip flow of simple fluids. Phys Rev E 81:011606. https://doi.org/10.1103/PhysRevE. 81.011606

15. Jabbarzadeh A (2013) Effect of nano-patterning on oleophobic properties of a surface. Soft Matter 9:11598-11608. https://doi.org/10.1039/C3SM52207E

16. Wang X, Zhao X, Jing C, Tao H, Han J (2005) Effects of nitric acid concentration on the stability of alumina sols. J Wuhan Univ Technol Mater Sci Ed 21(1):102-125

17. Verho T, Bower C, Andrew P, Franssila S, Ikkala O, Ras R (2011) Mechanically durable superhydrophobic surfaces. Adv Mater 23(5):673-678

18. Bassani R, Piccigallo B (1992) Hydrostatic lubrication. Elsevier, Amsterdam. ISBN $044488498 x$

19. Szeri A (1998) Fluid film lubrication. Cambridge University Press, Cambridge U.K. ISBN 0521481007

20. Hamrock B, Schmid S, Jacobson B (2004) Fundamentals of fluid film lubrication, 2nd edn. Marcel Dekker, Inc., New York. ISBN 0-8247-5371-2

21. Rowe W (2012) Hydrostatic, aerostatic and hybrid bearing design. Elsevier, Oxford. ISBN: 978-0-12-396994-1

22. Martell M, Rothstein J, Perot J (2010) Phys Fluids 22. https://doi.org/10.1063/1.3432514

23. Daub C, Wang J, Kudesia S, Bratko D, Luzar A (2010) The influence of molecular-scale roughness on the surface spreading of an aqueous nanodrop. Faraday Discuss 146:67-77. https://doi.org/10.1039/B927061M

24. Motta A, Cannelli O, Boccia A, Zanoni R, Raimondo M, Caldarelli A, Veronesi F (2015) A mechanistic explanation of the peculiar amphiphobic properties of hybrid organic-inorganic coatings by combining XPS characterization and DFT modeling. ACS Appl Mater Interfaces 7:19941-19947. https://doi.org/10.1021/acsami.5b04376

25. Malavasi F, Veronesi A, Caldarelli M, Zani M, Raimondo M Marengo (2016) Is a knowledge of surface topology and contact angles enough to define the drop impact outcome? Langmuir 32(25):6255-6262. https://doi.org/10.1021/acs.langmuir.6b01117

26. Raimondo M, Veronesi F, Boveri G, Guarini G, Motta A, Zanoni R (2017) Superhydrophobic properties induced by sol-gel routes on copper surfaces. Appl Surf Sci 422:1022-1029. https:// doi.org/10.1016/j.apsusc.2017.05.257

27. Raimondo M, Blosi M, Caldarelli A, Guarini G, Veronesi F (2014) Wetting behavior and remarkable durability of amphiphobic aluminum alloys surfaces in a wide range of environmental conditions. Chem Eng J 258:101-109. https://doi.org/10.1016/j.cej.2014.07.076 
28. Caldarelli A, Raimondo M, Veronesi F, Boveri G, Guarini G (2015) Sol-gel route for the building up of superhydrophobic nanostructured hybrid-coatings on copper surfaces. Surf Coat Technol 276:408-415. https://doi.org/10.1016/j.surfcoat.2015.06.037

29. Rizzo G, Bonanno A, Massarotti G, Paoluzzi R, Raimondo M, Blosi M, Veronesi F, Caldarelli A, Guarini G (2015) Axial piston pumps slippers with nanocoated surfaces to reduce friction. Int J Fluid Power 16(1):1-10. https://doi.org/10.1080/14399776.2015.1006979

30. Bonanno A, Masarotti G, Rizzo G, Paoluzzi R, Raimondo M, Blosi M, Veronesi F, Caldarelli A (2014) Application of nanostructured layer to improve the Energy efficiency in hydraulic piston pump. In: Proceedings of the 9th JFPS international symposium on fluid power, Matsue, October 28-31. ISBN 4-931070-10-8

31. Rizzo G, Bonanno A, Massarotti G, Pastorello L, Raimondo M, Veronesi F, Blosi M (2016) Energy efficiency improvement by the application of nanostructured coatings on axial piston pump slippers. In: 10th international fluid power conference I Dresden, Group 5-Pumps I Paper 5-6, pp 313-328

32. Terkaj W, Tolio T (2019) The Italian flagship project: factories of the future. In: Tolio T, Copani G, Terkaj W (eds) Factories of the future. Springer

Open Access This book is licensed under the terms of the Creative Commons Attribution 4.0 International License (http://creativecommons.org/licenses/by/4.0/), which permits use, sharing, adaptation, distribution and reproduction in any medium or format, as long as you give appropriate credit to the original author(s) and the source, provide a link to the Creative Commons licence and indicate if changes were made.

The images or other third party material in this book are included in the book's Creative Commons licence, unless indicated otherwise in a credit line to the material. If material is not included in the book's Creative Commons licence and your intended use is not permitted by statutory regulation or exceeds the permitted use, you will need to obtain permission directly from the copyright holder. 